Nervenarzt 2021 · 92:935-940

https://doi.org/10.1007/s00115-021-01136-7

Angenommen: 18. April 2021

Online publiziert: 27. Mai 2021

(c) Springer Medizin Verlag $\mathrm{GmbH}$, ein Teil von Springer Nature 2021

\section{Die Unterbringung von Menschen mit psychischen Störungen in ge- schlossenen Wohnheimen kann unseres Erachtens nur eine zeit- lich befristete Ultima Ratio, d. $h$. ein Mittel der letzten Wahl, für wenige Einzelfälle mit klar ersicht- lichem Zeithorizont darstellen. Die heterogene und intransparente Heimversorgungsstruktur vor al- lem im Hinblick auf die Versorgung von Menschen mit schweren psychi- schen Erkrankungen in Deutschland folgt diesem Prinzip nicht. Viel- mehr nimmt sie, aufgrund fehlender Regelungen und einem daraus resultierenden Mangel an Alterna- tiven im gemeindepsychiatrischen Versorgungssystem, oft eine Lücken- büßerfunktion ein, die auch von Seiten der Leistungserbringer $\mathbf{z}$. T. mit Nachdruck eingefordert wird.}

\section{Hintergrund}

Die vorliegende Arbeit geht von der Prämisse aus, dass es aufgrund psychischer Erkrankungen ein chronisch selbstgefährdendes Verhalten geben kann, das darüber hinaus auch mit Fremdgefährdung einhergehen kann und das einer therapeutischen Perspektive bedarf, um

\author{
R. Borbé ${ }^{1,2} \cdot$ M. Rosemann ${ }^{3} \cdot$ A. Nienaber ${ }^{4} \cdot$ I. Steinhart ${ }^{5,6} \cdot$ A. Heinz \\ ${ }^{1}$ Klinik I für Psychiatrie und Psychotherapie, Universität Ulm, Ulm, Deutschland \\ ${ }^{2}$ Zentren für Psychiatrie Südwürttemberg, Ravensburg-Weissenau, Deutschland \\ ${ }^{3}$ Träger gGmbH Berlin, Berlin, Deutschland \\ ${ }^{4}$ Fachhochschule Münster, Münster, Deutschland \\ ${ }^{5}$ v. Bodelschwinghsche Stiftungen Bethel, Bethel, Deutschland \\ ${ }^{6}$ Institut für Spzialpsychiatrie Mecklenburg-Vorpommern, Greifswald, Deutschland \\ ${ }^{7}$ Klinik für Psychiatrie und Psychotherapie, Charité Universitätsmedizin Berlin, Berlin, Deutschland
}

\title{
Gemeindepsychiatrische Ansätze zur Vermeidung der Unterbringung in geschlossenen Heimen - eine Positionsbestimmung
}

die betroffenen Personen selbst und gegebenenfalls zeitweise auch ihre Umwelt zu schützen. Besonders schwierig für die gemeindepsychiatrische Versorgung sind beispielsweise Situationen, in denen chronisch psychotisch erkrankte Personen aus Angst und zur Abwehr ihres Verfolgungserlebens Gegenstände in Brand setzen oder andere gefährliche Handlungen ausführen. Diese Subgruppe innerhalb der gemeindepsychiatrischen Patient*innen benötigt aufgrund einer (anhaltenden) Symptomschwere in Verbindung u.a. mit Suchterkrankungen und/oder komplexen sozialen Problemen eine ebenso komplexe und langfristige Unterstützungs- und $\mathrm{Be}$ handlungsperspektive, in Einzelfällen und zeitlich befristet auch in einer geschlossenen Unterbringung.

Demgegenüber gibt es in Deutschland nach wie vor eine größere Gruppe von Patient ${ }^{*}$ innen, die in geschlossenen Einrichtungen nach $\$ 1906$ Bürgerliches Gesetzbuch (BGB) untergebracht und dort fehlplatziert sind [5, 16, 17]. Um wie viele Betroffene es sich dabei handelt, ist bundesweit nicht bekannt und kann nur geschätzt werden [17]. Zudem ist davon auszugehen, dass ein Teil dieser Patient ${ }^{*}$ innengruppe auch im Sinne einer Transinstitutionalisierung in einer fo- rensischen Klinik untergebracht ist, sich in Haft befindet [12] oder in Obdachlosigkeit lebt [15], was die Schätzung zusätzlich erschwert. Auch über die Dauer einer solchen geschlossenen Unterbringung in einem Heim liegen zwar Schätzungen, aber keine bundesweiten Zahlen vor [17].

International findet sich eine gute Evidenz für multidisziplinäre, flexible gemeindepsychiatrische Teams, die Alternativen zur geschlossenen Unterbringung bieten könnten [10]. Diese Teams sind niederschwellig erreichbar, koordinierend und settingübergreifend, d.h. sowohl aufsuchend als auch stationär tätig.

Die S3-Leitlinie „Psychosoziale Therapien bei schweren psychischen Erkrankungen" der Deutschen Gesellschaft für Psychiatrie und Psychotherapie, Psychosomatik und Nervenheilkunde (DGPPN) geht in dieselbe Richtung:

In allen Versorgungsregionen soll eine gemeindepsychiatrische, teambasierte und multiprofessionelle Behandlung zur Versorgung von Menschen mit schwerer psychischer Erkrankung zur Verfügung stehen (Empfehlungsgrad A; [4]).

Vor diesem Hintergrund befasst sich die vorliegende Positionsbestimmung 
mit der Frage, welche Interventionen in Deutschland zur Verfügung stehen, um eine bedarfsgerechte Versorgung der betroffenen Personen zu gewährleisten. Fehlplatzierungen, beispielsweise junger Psychosepatient ${ }^{*}$ innen in Pflegeeinrichtungen nach Sozialgesetzbuch (SGB) XI, und geschlossene Unterbringungen der Betroffenen könnten so vermieden werden. Hiermit wären die derzeitigen zu hohen Unterbringungsraten $\mathrm{zu}$ verringern und die Schaffung neuer Kapazitäten zu verhindern.

\section{Methodik}

Auf Initiative der DGPPN wurde eine Taskforce "Geschlossene Heime“ ins Leben gerufen mit dem Ziel, sich dem Thema aus Sicht der Fachgesellschaft $\mathrm{zu}$ widmen und einen offensichtlich dringend notwendigen Diskurs, unter Berücksichtigung einschlägiger Literatur, über das Thema anzuregen. Der Diskussionsprozess wurde in einer Experten*innengruppe trialogisch unter Beteiligung von Organisationen der Betroffenen und Angehörigen geführt, die Ergebnisse aber nicht formal konsentiert.

\section{Ergebnisse}

In der nationalen und internationalen Literatur fanden sich keine Publikationen, in denen als Intervention ein alternatives Versorgungsangebot mit einer Unterbringung in einem geschlossenen Wohnheim verglichen wird. Die Literatur zur Versorgung von Menschen mit schweren psychischen Erkrankungen verweist auf teambasierte und multiprofessionelle Intensivbetreuung im Sinne von "assertive community treatment" (ACT) oder „intensive case management" (ICM) im häuslichen Umfeld oder unterstütztes (betreutes) Wohnen („supported housing“; [10]).

Diese Empfehlungen decken sich mit den grundlegenden Empfehlungen für die gemeindepsychiatrische Versorgung aus Leitlinien [4] oder dem personenzentrierten Ansatz der Aktion Psychisch Kranke (APK; [8]). Geschlossene Heime finden keine oder nur eine kurze Erwähnung. Auch in der neuen Nomenklatur infolge der Neufassung des SGB IX gibt es den Begriff Heime nicht mehr. Jegliche stationären Wohnformen gelten nun als besondere Wohnformen. Aus Gründen der Verständlichkeit und Klarheit, wird in diesem Artikel weiterhin der Begriff „Heim" verwendet.

\section{Geschlossene Unterbringung}

Die deutsche Versorgungslandschaft kennt verschiedene Einrichtungsarten, in denen eine Unterbringung nach $\$ 1906$ BGB vollzogen wird. Die häufigsten sind, neben psychiatrischen Krankenhäusern, Heime und in kleinerer Zahl therapeutische Wohngruppen (TWG), die als gesamte Einrichtung oder für einen Teilbereich der Einrichtungen die Freiheitsentziehung möglich machen.

Therapeutische Wohngruppen haben das Ziel, dass Betroffene nach der meist befristeten Maßnahme, in einer weniger intensiv betreuten Wohnform leben können. Die Überleitung bzw. Entlassung aus einer solchen Maßnahme führt aber einerseits oft $\mathrm{zu}$ einem Beziehungsabbruch, andererseits ist die Stufe zur weniger intensiven Betreuungsform meist sehr hoch.

Die sehr heterogen gearteten fakultativ geschlossenen Heime weisen große strukturelle Unterschiede auf, z.B. in der Platzzahl, der personellen Besetzung oder auch der Fachlichkeit. Viele dieser Einrichtungen sind nicht Teil einer Verbundstruktur (z.B. eines Gemeindepsychiatrischen Verbundes), nehmen überregional auf und verfolgen keinen spezifischen psychiatrischen Auftrag [17]. Zunehmend ist bundesweit ein Trend $\mathrm{zu}$ beobachten, dass die überregionalen Aufnahmen begrenzt werden. Die Folge sind u.a. verlängerte Liegedauern in den psychiatrischen Kliniken bei fehlender Anschlussversorgung und Entlassung in die Obdachlosigkeit.

\section{Geschlossene Unterbringung vermeiden}

Im Folgenden geht es darum zu beschreiben, wie sich die Wahrscheinlichkeit der Unterbringung in einer geschlossenen Wohnform ausschließen oder zumindest verringern lässt. Die Lösungsan- sätze werden anhand von vier Punkten beschrieben:

\section{Akutstationäre Versorgung durch psychiatrische Kliniken und Abteilungen (1)}

Die Überweisung in geschlossene Versorgungsangebote erfolgt in den meisten Fällen aus dem Bereich der akutstationären Versorgung [17]. Vor diesem Hintergrund müssen erste Ansätze zur Vermeidung bereits im Bereich der psychiatrischen Kliniken und Abteilungen ansetzen. Das könnten z. B. längerfristige und ggf. intermittierende Behandlungen der betreffenden Personen sein, aber auch komplexe Konzepte wie z.B. das Weddinger Modell [9], die unterschiedliche Maßnahmen integrieren.

\section{》) Das Weddinger Modell verfolgt die konsequente Einbeziehung von Peers}

Ausgangspunkt für das Weddinger Modell war die Entwicklung einer offenen, Recovery-orientierten Akutpsychiatrie. Der Ansatz verfolgt den Einbezug der Betroffenen und ihres Umfelds durch Behandlungskontinuität, völlige Transparenz der therapeutischen Planung und der konsequenten Einbeziehung von Peers. Damit kann dieses Modell auch im Sinne eines präventiven Ansatzes einer Unterbringung in einer geschlossenen Wohnform im Anschluss an den stationären Krankenhausaufenthalt vorbeugen. Durch die Fokussierung auf Ressourcen und Strategien der $\mathrm{Pa}$ tient ${ }^{*}$ innen gelingen Motivationsförderung und Besserung der Resilienz und eine Förderung der Selbstachtung und Resistenz. Die Institution muss sich auf die besondere Situation des Betroffenen einstellen und die aus Sicht der $\mathrm{Pa}$ tient*innen vorhandenen Barrieren für die Annahme von Unterstützung und/ oder Behandlung abbauen.

\section{Gemeindepsychiatrische Versorgung (2)}

Gemeindepsychiatrische Verbünde (GPV) ermöglichen die leistungserbringerübergreifende Koordination von Unterstützungsleistungen und eine gemeinsame 
Nervenarzt 2021 · 92:935-940 https://doi.org/10.1007/s00115-021-01136-7

(c) Springer Medizin Verlag GmbH, ein Teil von Springer Nature 2021

R. Borbé · M. Rosemann · A. Nienaber · I. Steinhart · A. Heinz

\section{Gemeindepsychiatrische Ansätze zur Vermeidung der Unterbringung in geschlossenen Heimen - eine Positionsbestimmung}

\section{Zusammenfassung}

Hintergrund. Die Unterbringung von Menschen mit psychischen Störungen in geschlossenen Heimen ist in vielen Fällen Ausdruck mangelnder alternativer Versorgungsangebote. In dem Spannungsfeld zwischen dem Bedürfnis nach Sicherung bei chronisch selbstgefährdendem Verhalten, rezidivierender akutpsychiatrischer Krisenintervention und mangelnder Perspektive, eine dauerhafte Therapie zu etablieren, wird vielerorts eine betreuungsrechtlich genehmigte Unterbringung in einem geschlossenen Heim durchgeführt.

Fragestellung. Welche Alternativen zur geschlossenen Heimunterbringung im gemeindepsychiatrischen Versorgungssystem gibt es in Deutschland?

\author{
Material und Methode. Unter Berücksich- \\ tigung einschlägiger Literatur wurde ein \\ Diskussionsprozess in einer Expertengruppe \\ trialogisch unter Beteiligung von Organisa- \\ tionen der Betroffenen und Angehörigen \\ durchgeführt. \\ Ergebnisse. Das gemeindepsychiatrische \\ Versorgungssystem in Deutschland ist \\ extrem heterogen. Das zergliederte so- \\ zialrechtliche und Finanzierungssystem \\ erschwert eine sektorenübergreifende und \\ kontinuierliche Versorgungsplanung und \\ Leistungserbringung. Passgenaue und \\ individuelle Leistungen, die eine geschlossene \\ Heimunterbringung der betroffenen Personen \\ verhindern könnten, gibt es in Deutschland \\ nur an wenigen Standorten und in Form \\ einzelner Modellprojekte.
}

Schlussfolgerungen. Die angesprochenen strukturellen und leistungsrechtlichen Defizite erfordern eine Reform der Rahmenbedingungen und ein Umdenken aller beteiligten Akteure, einschließlich der Kliniken. Alternative Ansätze zur Versorgung von Menschen mit schweren psychischen Störungen werden skizziert, darunter das Weddinger Modell, verbindliche gemeindepsychiatrische Strukturen, das funktionale Basismodell und Assistenzleistungen im Sozialgesetzbuch (SGB) IX infolge der Neuregelungen des Bundesteilhabegesetzes (BTHG).

\section{Schlüsselwörter}

Fallkonferenzen - Versorgungsangebote . Steuerung · Personenzentrierter Ansatz . Assistenzleistungen

\section{Community mental health approaches for avoidance of patient placement in closed psychiatric facilities-a positioning}

\section{Abstract}

Background. In many cases the placement of people with mental disorders in closed residential homes is an expression of the lack of alternative care options. In the area of tension between the need for security in the case of chronically self-endangering behavior, recurrent acute psychiatric crisis interventions and a lack of perspective to establish a permanent therapy, in many places a placement in a closed facility approved under care law is carried out.

Objective. What alternatives are there to closed institutional care in the psychiatric care system in Germany?

Methods. A trialogical discussion process was carried out in an expert panel taking the relevant literature into consideration and with the participation of organizations of those affected and their relatives.

Results. The community psychiatric care system in Germany is extremely heterogeneous. The fragmented sociolegal and financing systems makes cross-sectoral and continuous care planning and service provision difficult. Precisely tailored and individualized services that could prevent the persons concerned from being placed in a closed home exist in Germany only at a few locations and in the form of individual model projects.

Conclusion. The structural and sociolegal deficits addressed require a reform of the institutional framework and a redirection of all actors involved, including the clinics. Alternative approaches to the care of people with severe mental disorders are outlined. These include the Wedding model, binding community psychiatric structures, the basic functional model and assistance services under the German Social Code IX following the revision of the Federal Participation Act.

Keywords

Case conference - Care options - Control . Person-centered approach · Assistance services
Versorgungsplanung in einer Region, meist unter Moderation der Kostenträger. Die Leistungserbringer gehen eine Versorgungsverpflichtung für die Region, meist einen Landkreis, ein. Wichtigstes Instrument war bisher die Hilfeplankonferenz, die in Zukunft, dem neuen Eingliederungshilferecht im SGB IX folgend, als Teilhabe- bzw. Gesamtplankonferenz primär durch den Kostenträger initiiert wird.
Erfahrungen aus dem Projekt „Vermeidung von Zwangsmaßnahmen im psychiatrischen Hilfesystem (ZVP)“ der Bundesarbeitsgemeinschaft Gemeindepsychiatrischer Verbünde e.V., das in den Jahren 2016 bis 2019 vom Bundesministerium für Gesundheit gefördert wurde, ergeben einige Anhaltspunkte für erfolgreiche Strategien zur Vermeidung oder wenigstens Verminderung der freiheitsentziehenden Unterbringungen in Heimen [13]. So haben sich insbeson- dere Konferenzen bewährt, in denen einrichtungsübergreifend und einzelfallbezogen Anlass- und Lebenssituationen von Menschen intensiv beraten werden, bei denen eine freiheitsentziehende Unterbringung erwogen oder deren richterliche Genehmigung schon beantragt worden sind. In diesen intensiven Fallbesprechungen, für deren Durchführung entsprechende Empfehlungen entwickelt worden sind, konnten in Einzelfällen alternative Unterstützungsangebote $\mathrm{zu}$ 
der beabsichtigten Maßnahme erörtert und entwickelt werden, v. a. in Stuttgart, Mönchengladbach, Viersen, Berlin [13].

\section{) In Einzelfallbesprechungen werden alternative Unterstüt- zungsangebote entwickelt}

In Berlin bestehen in jedem Versorgungsbezirk sog. „Steuerungsgremien Psychiatrie“, an denen alle Anbieter therapeutischer Wohnformen verpflichtend teilnehmen, damit allen Patient ${ }^{*}$ innen poststationär ein ihren persönlichen Bedürfnissen und Bedarfen angepasstes Versorgungsangebot gemacht werden kann.

\section{Integrierende Konzepte der Versorgung (3)}

Das zergliederte Versorgungssystem mit den unterschiedlichen Zuständigkeiten führt zu Brüchen in der Versorgung und verhindert die, im Hinblick auf die hier in den Blick genommene Zielgruppe, so wichtige Kontinuität. Integrierte Leistungsangebote, die eine passgenaue und kontinuierliche Leistungsplanung und -erbringung, auch über verschiedene Settings und Sozialgesetzbücher hinweg ermöglichen, stellen hier eine Lösung dar.

Das funktionale Basismodell zur gemeindepsychiatrischen Versorgung schwer psychisch kranker Menschen beschreibt konsequent Funktionen, die das Versorgungssystem vorhalten muss, um eine umfassende und flexible Versorgung zu gewährleisten [18, 20]. Das Modell löst sich dadurch von einer institutionszentrierten und finanzierungsbasierten Sichtweise, die bei der Planung von Versorgungsangeboten häufig dazu führt, dass passgenaue Angebote nicht realisiert werden können, weil sie entweder nicht vorhanden oder nicht finanzierbar sind. Die Steuerung erfolgt durch die betroffene Person mit Unterstützung eines multiprofessionellen Teams. Durch die funktionale Beschreibung erfolgt auch keine Festlegung darauf, wer z.B. die Steuerungsfunktion übernimmt. Dies kann sowohl ein multiprofessionelles Team mit Bezugsperson eines sozialpsychiatrischen Trägers sein als auch der niedergelassene Facharzt mit seinem Team.

Regionalbudgets im SGB $V$ ermöglichen eine sehr differenzierte Fallsteuerung. Vor allem die Möglichkeit nachgehender, aufsuchender Behandlung in flexibler Intensität kommt nahe an das Idealmodell des „flexible assertive community treatment" (FACT; [19]) heran. Das Team, das die Langzeitbetreuung chronisch psychisch Kranker in der Gemeinde gewährleistet, ist in der Lage, bei Bedarf auch wie ein Krisenteam zu arbeiten und vermeidet dadurch Beziehungsabbrüche und stationäre Akutinterventionen im Sinne eines „No-drop-out“Ansatzes [11]. In Deutschland sind Modellvorhaben zum Regionalbudget aber im Wesentlichen auf Norddeutschland beschränkt [3].

\section{》) Assistenzleistungen ermög- lichen wohnformunabhängige Betreuungsangebote}

Eine weitere Möglichkeit der Verbesserung liegt in den Assistenzleistungen im SGB IX. Assistenzleistungen nach $\$ 78$ SGB IX dienen der „selbstbestimmten und eigenständigen Bewältigung des Alltags, einschließlich der Tagesstrukturierung“. Assistenzleistungen ermöglichen wohnformunabhängige hochintensive Betreuungsangebote. Diese können aufgrund der hohen Betreuungsintensität, die notwendig sein kann, mit erheblichen Mehrkosten verbunden sein. Sie sind universell einsetzbar, d.h. sie können sogar bei Obdachlosigkeit erbracht werden [6]. Das funktionale Basismodell sieht vor, eine koordinierende Bezugsperson mit Blick auf die Assistenzleistung der persönlichen Lebensplanung zu finanzieren [7].

\section{Monitoring und Evaluation (4)}

Zwingend erforderlich sind darüber hinaus Strukturen, die ermöglichen, dass eine geschlossene Unterbringung für die Betroffenen immer nur ein zeitlich befristeter Übergangsstatus ist und während der Unterbringung ständig nach offenen Arrangements gesucht wird. Die Grundlage der Versorgung muss eine klare und unmissverständliche Sozialraum- orientierung („catchment area“) sein, mit einer bedarfsbezogenen nach oben offenen Einzelfallfinanzierung anstelle der geschlossenen Unterbringung sowie eine kontinuierliche Integration von Behandlung in die geschlossene Unterbringung in einer besonderen Wohnform mit dem Ziel der Inklusion und der Teilhabe [2].

\section{Diskussion}

Die Unterstützung und Behandlung von Menschen mit schweren psychischen Erkrankungen im Sinne einer modernen und gemeindepsychiatrischen Versorgung erfordert ein differenziertes, sektorübergreifendes, multiprofessionelles und im Hinblick auf das Setting flexibles Behandlungsangebot, um eine personenzentrierte Behandlung $\mathrm{zu}$ ermöglichen.

\section{Geschlossene Unterbringung nur als Ultima Ratio}

Ist eine freiheitsentziehende Unterbringung im gut begründeten Einzelfall bei der oben beschriebenen, multimorbiden Patient ${ }^{*}$ innengruppe zur Vermeidung langfristiger Selbstschädigung nicht zu vermeiden, muss sie zwingend zeitlich befristet, regelhaft geprüft und im Hinblick auf eine therapeutische Strategie, die die Inklusion in die Gemeinde zum Ziel hat, geplant und evaluiert werden. Im Hinblick auf ein Monitoring ist es erforderlich, dass die Anzahl der geschlossenen Heimplätze bundesweit erfasst und transparent berichtet wird. Dies gilt auch für die oben ausgeführten alternativen Versorgungsformen, die längst nicht flächendeckend etabliert sind und mit erheblichen Implementierungshindernissen wie z.B. Partikularinteressen einzelner Stakeholder zu kämpfen haben, zuvorderst einer Unterfinanzierung erforderlicher Maßnahmen.

\section{Kliniken in der Pflicht}

Die Kliniken haben es in der Hand, ihre gemeindepsychiatrische Arbeit zu stärken, die, frei nach Rössler, mit der Aufnahme beginnt [14]. Im Hinblick auf ihre Behandlungskonzepte können sie auf evidenzbasierte Programme wie z. B. das 
Weddinger Modell zurückgreifen, um eine individuelle, personenzentrierte $\mathrm{Be}$ handlung für die Betroffenen zu ermöglichen und alles dafür zu tun, geschlossene Unterbringungen $\mathrm{zu}$ vermeiden. Bei Patient ${ }^{*}$ innen mit krankheitsbedingter, chronischer Selbstgefährdung ist aufsuchendes Arbeiten z. B. im Rahmen aufsuchender PIA(psychiatrische Institutsambulanz)-Arbeit oder stationsäquivalenter Behandlung (StäB) hilfreich, um krankheitsfördernde Faktoren im direkten Lebensumfeld einschätzen zu können.

\section{Gemeinsame Verantwortung gemeindepsychiatrischer Leistungserbringer}

Die bundesweite Etablierung von GPV oder anders gearteter gemeindepsychiatrischer Verbundstrukturen ist ein wichtiger Schritt, um die Hilfeplanung zu harmonisieren und transparenter zu gestalten. Anders ist unseres Erachtens die Umsetzung von Artikel 19 der UN-Behindertenrechtskonvention (UN-BRK), der u. a. die selbstbestimmte Lebensführung und die gleichberechtigte Wahl des Aufenthaltsortes beinhaltet, nicht zu realisieren [1]. Das heißt auch, dass die Kostenträger bereit sein müssen, den Betroffenen bei seinem Wunsch zu unterstützen, den Wohnort zu wechseln.

Strukturen wie Gemeindepsychiatrische Verbünde können dazu beitragen, eine Praxis einzudämmen, die mit Artikel 19 UN-BRK nicht vereinbar ist: die Verbringung untergebrachter psychisch Kranker in wohnortferne Heime, teils quer durch Deutschland. Sowohl im Artikel 19 UN-BRK [1] als auch im $\$ 104$ Abs. 3 SGB IX [2] ist festgelegt, dass Menschen mit Behinderungen selbst entscheiden können, wo und mit wem sie leben, und nicht verpflichtet sind, besondere Wohnformen zu wählen.

\section{Höhere Kosten können ethisch geboten sein}

Gemeindepsychiatrische Strukturen, die eine geschlossene Unterbringung in einem Heim deutlich verringern können oder gar überflüssig machen, bedürfen verlässlicher Strukturen, wie einem GPV, einer zentralen Steuerung, wie im funktionalen Basismodell skizziert, und vor allem einer flexiblen Finanzierung. Diese wird auch durch Regionalbudgets erreicht, die ggf. sozialgesetzbuchübergreifend gestaltet sein müssen, oder durch eine konsequente Umsetzung der im SGB IX beschriebenen Teilhabeleistungen, vor allem der Assistenzleistung. Dies kann in Einzelfällen, bei sehr personalintensiven Einzelarrangements wie z.B. 1:1-, 2:1- oder auch 3:1-Betreuungen, zu insgesamt hohen Fallkosten führen. Stattdessen die günstigere Heimunterbringung $\mathrm{zu}$ wählen widerspricht der UN-BRK, ist unethisch und auf lange Sicht wahrscheinlich kostenintensiver.

\section{Fazit für die Praxis}

- Unterbringungen aufgrund selbstgefährdenden Verhaltens müssen als Ultima Ratio zeitlich befristet sein.

- Kliniken müssen sich als Teil des gemeindepsychiatrischen Versorgungssystems verstehen und die komplexe Hilfeplanung trialogisch und transparent gestalten.

- Gelingende Alternativlösungen sollten innerhalb der Versorgungsregionen gesucht werden und müssen integraler Bestandteil des Pflichtversorgungsangebots sein.

- Individuelle, personenzentrierte Alternativen zur geschlossenen Unterbringung erfordern eine hohe Flexibilität aller Beteiligten.

- Assistenzleistungen nach Sozialgesetzbuch (SGB) IX ermöglichen eine wohnformunabhängige Unterstützung.

\section{Korrespondenzadresse}

\section{Dr. R. Borbé, MHBA}

Zentren für Psychiatrie Südwürttemberg Weingartshoferstr. 2, 88214 Ravensburg-

Weissenau, Deutschland

raoul.borbe@zfp-zentrum.de

\section{Einhaltung ethischer Richtlinien}

Interessenkonflikt. R. Borbé, M. Rosemann, A. Nienaber, I. Steinhart und A. Heinz geben an, dass kein Interessenkonflikt besteht.
Für diesen Beitrag wurden von den Autoren keine Studien an Menschen oder Tieren durchgeführt. Für die aufgeführten Studien gelten die jeweils dort angegebenen ethischen Richtlinien.

\section{Literatur}

1. Bundesgesetzblatt (2008) Gesetz zu dem Übereinkommen der Vereinten Nationen vom 13. Dezember 2006 über die Rechte von Menschen mit Behinderungen sowie zu dem Fakultativprotokoll vom 13. Dezember 2006 zum Übereinkommen der Vereinten Nationen über die Rechte von Menschen mit Behinderungen. . In:Bundesgesetzblatt, Bonn p 1419-1457

2. Bundesgesetzblatt (2016) Gesetz zur Stärkung der Teilhabe und Selbstbestimmung von Menschen mit Behinderung (Bundesteilhabegesetz-BTHG). In:Bundesgesetzblatt, Bonn, p 3234-3340

3. Deister A, Zeichner D, Witt T et al (2010) Veränderung der psychiatrischen Versorgung durch ein Regionales Budget. Psychiat Prax 37:335-342

4. GühneU,WeinmannS, Riedel-Heller SGetal (2018) S3-Leitlinie Psychosoziale Therapien bei schweren psychischen Erkrankungen. Springer, Berlin

5. Jenderny S, Schreiter J, Steinhart I (2020) Psychiatrische Wohnheime in Deutschland Transparenz und Strukturen. Psychiatr Prax 47:260-266

6. Konrad M (2019) Die Assistenzleistung: Anforderungen an die Eingliederungshilfe durch das BTHG. Psychiatrie Verlag,

7. Konrad M, Rosemann M (2016) Betreutes Wohnen: mobile Unterstützung zur Teilhabe. Psychiatrie Verlag,

8. Kunze $H$ (1995) Personenzentrierte Orientierung der Hilfen. In: Kruckenberg P, Jagoda B, (APK) APK (Hrsg) Personalbemessung im komplementären Bereich - von der institutions- zur personenbezogenen Behandlung und Rehabilitation. Aktion Psychisch Kranke, Bonn, S95-107

9. Mahler L, Heinz A, Jarchov-Jàdi I et al (2019) Therapeutische Haltung und Strukturen in der (offenen) Akutpsychiatrie: Das Weddinger Modell. Nervenarzt 90:700-704

10. National Institute for Health and Care Excellence (NICE) (2020) Psychosis and schizophrenia in adults: prevention and management

11. Nielsen CM, Hjorthøj C, Killaspy H et al (2021) The effect of flexible assertive community treatment in Denmark: a quasi-experimental controlled study. Lancet Psychiatry 8:27-35

12. Priebe S, Badesconyi A, Fioritti A et al (2005) Reinstitutionalisation in mental health care: comparison of data on service provision from six European countries. BMJ 330:123-126

13. Rosemann M, Gallinat J, Heinz A etal (2020) Vermeidung von Zwangsmaßnahmen im psychiatrischen Hilfesystem. In:Bundesarbeitsgemeinschaft Gemeindepsychiatrischer Verbünde e. V. (BAG GPV). https://www.bag-gpv.de/fileadmin/downloads/ ZVP_Gesamtbericht_final.pdf. Zugegriffen: 27. Mai 2021

14. Rössler W, Lauber C (2004) Psychiatrische Rehabilitation - Vom Behinderungsmodell zum Empowerment. In: Psychiatrische Rehabilitation. Springer, Berlin Heidelberg, S 1-4

15. Schreiter S, Gutwinski S, Rössler W (2020) Wohnungslosigkeit und seelische Erkrankungen. Nervenarzt 91:1025-1031 
16. Steinhart I (2018) Schwer psychisch krank in Deutschland - (k) ein "gutes Leben". Psychiat Prax 45:341-343

17. Steinhartl, Jenderny S, Schreiter J (2020) (Geschlossene) besondere Wohnformen als unverzichtbarer Teil der regionalen Verbundstrukturen in Deutschland? Psychiat Prax 47:370-375

18. Steinhart I, Wienberg G (2016) Das Funktionale Basismodell für die gemeindepsychiatrische Versorgung schwer psychisch kranker Menschen - Mindeststandard für Behandlung und Teilhabe. Psychiat Prax 43:65-68

19. Van Veldhuizen JR (2007) FACT: a Dutch version of ACT. Community Ment Health J43:421-433

20. Wienberg G, Steinhart I (2020) Das Funktionale Basismodell der Versorgung von Menschen mit schweren psychischen Erkrankungen-ein Update. Psychiat Prax 47:9-15
Heinrich Berger, Peter Bechmann, Véronique Dehimi (Hrsg.)

\section{Psycho-Tisch}

Geschichten und Bilder aus dem Münchner Psychose-Seminar

Pabst Science Publishers 2020, 336 S., 30 EUR

"Psycho-Tisch" - so wie eine kleinste orthografische Veränderung des Titel-gebenden Wortes einen originell- kreativen Perspektivenwechsel bewirkt, so bietet das gesamte Buch dem Leser kreative, eigenwillige und konstruktive Wechsel-Perspektiven auf das Phänomen „psychotisch“ resp. das Psychotisch-sein. Dies gilt für die anregende Form-Gestaltung mit vielen Bildern und Fotographien von Künstlerinnen und Künstlern, allesamt Mitglieder/Teilnehmer des Münchner Psychose-Seminars wie für die aufgetischten Text-Beiträge der 37 Autoren.

Entstanden ist ein sehr bemerkenswertes, spannendes Lesebuch, in dem vor allem anderen die Vielfalt individueller Lebenssituationen und Biografien sowie das persönliche Erleben von Menschen mit einer Psychose-Erfahrung den Tenor bestimmen. Dass es neben dem Bekenntnis zum Grundsatz "es ist normal verschieden zu sein" dabei auch um eine krankhafte Störung mit viel persönlicher Leid-Erfahrung, um besorgte bis verzweifelte Angehörige geht, kommt ebenso auf den Tisch wie der sehr ernsthafte Ansatz psychiatrischer und psychotherapeutischer Hilfe. Berichtet wird aus subjektiver Perspektive vom Erleben, vom "ganz normalen Wahnsinn", von der Suche nach dem Sinn des Ganzen und von den Brücken zu Wissenschaft und Gesellschaft. In Geschichten, Gedichten und Berichten zur Person und „zur Sache“ kommen konkret zur Sprache: die heilende Wirkung des Schreibens, die dramatischen Erfahrungen „aus dem Auge des Orkans" von Psychose-Erfahrenen, die Dünnhäutigkeit, das innere Panik-Orchester, das Sich-selbst-zerlegen, das Piepen des Geheimdienstes, der Wahn, das Glück und die Traurigkeit. Und zwischendrin immer wieder anregende oder „anfassende“ Bilder, Fotographien, Lyrik mit Tiefgang. Es ist gänzlich unmöglich, die Breite und Vielgestaltigkeit zu beschreiben; jede einzelne Seite des Buches regt an, verführt zum Weiterlesen und Blättern. Es geht um Dramen und Dilemmata, Überforderung und Selbstschutz, um Chancen für einen Neubeginn. Es finden sich ein krimi-spannender Trialog über eine Reise in der Psychose (nach Hongkong) und dann mit Höhen und Tiefen die Erfahrungen mit der psychiatrischen Behandlung, mit Fixierung, aber auch mit der Kunsttherapie und immer wieder die Behandlung mit Psychiatern, Psychologen, Pflegern. Ablehnung, Ambivalenz und Vertrauensaufbau heißen hier die Überschriften. Dass Medikamente als ein großes Themenfeld vielschichtig abgehandelt werden, wird niemand verwundern, der irgendwie schon mal Kontakt zur Psychiatrie hatte.

Die Expertenrolle ernst nehmen und Expertenwissen kritisch vermitteln, den Patienten empowern, mit behutsamen Worten die heilsame Wirkung suchen, Verständnisbrücken bauen zwischen Psychose-Erfahrenen und ihren Angehörigen und den Psychiatern / Therapeuten, so lautet das Mantra von Josef Bäuml, eines maßgeblichen Förderers des wegweisenden Münchner Psychose-Seminars.

Als Initiator und Protagonisten der Psychoedukationsbewegung in Deutschland seit den frühen 90er Jahren und als dichtender Sozialpsychiater an der TU München sieht er sich als Mitglied einer trialogischen Solidargemeinschaft, das ggf. auch eigene Hilflosigkeit sich einzugestehen traut. Bäuml unterstützt nachhaltig den Geist des respektvollen Austausches im PsychoseSeminar und er beschreibt die Synergien von Psychoedukation und Psychose-Seminar. Wenn der Psychotherapeut Heinrich Berger als Vertreter der trialogisch zusammengesetzten Herausgebergruppe das Buch nach 300 Seiten abschließt mit einem großen Dank-Gedicht an Dorothea Buck, die gemeinsam mit Thomas Bock das erste Hamburger Psychose-Seminar initiierte, dann ist damit die Aufbruch-Dimension des Buches nochmals umrissen. Das sehr gelungene Lese-, Bilder- und Nachdenk-Buch wird sicher seinen großen Leserkreis finden; die edle Aufmachung und Gestaltung passen zum edlen Inhalt. Das Buch ist ein trialogisches Referenzbuch; es sollte in jedem Wartezimmer eines Psychiaters und auf jeder psychiatrischen Station zur Standardlektüre gehören. 